\title{
Long-term evolution of radial surfaces of constant angular frequency
}

\author{
Dirk K. Callebaut ${ }^{1}$ and Valentin I. Makarov ${ }^{2}$ \\ ${ }^{1}$ Physics Department, CDE, University of Antwerp (UA), B-2610 Antwerp, Belgium, email: \\ Dirk.Callebaut@ua.ac.be \\ ${ }^{2}$ Main Astronomical Observatory, Pulkovo, St. Petersburg, 196140, Russia, email: \\ makarov@gao.spb.ru
}

\begin{abstract}
The radial surfaces of constant angular frequency may be connected with the upper rest latitude of the filament bands (or boundaries of large-scale unipolar magnetic regions) and with the separation of the sunspot region and the polar region (polar faculae, etc.). The equator-ward evolution of the rest latitudes observed since cycle 12 may be a forebode of a deep minimum.
\end{abstract}

\section{Introduction}

A filament band, consisting of a chain of filaments and filament channels, is the bordering region between two large-scale quasi-unipolar magnetic regions of low field strength (say a few gauss). These regions are generated near the equator and move to the poles, each according to its hemisphere. In times of low magnetic activity there are 2 filament bands in each hemisphere, but during active periods 3 or 4 may occur, besides the very vague one near the equator. However, during the minimum activity of the Sun, they stay around their 'rest latitude'. During cycle 12 this was $53^{\circ}\left(55^{\circ}\right.$ north and $51^{\circ}$ south $)$ for the older one (hence being already at a higher latitude) and $28^{\circ}\left(25^{\circ}\right.$ north and $31^{\circ}$ south) for the younger one (hence lower one). Since cycle 12 these 'rest latitudes' came steadily lower: $36^{\circ}$ (north) and $39^{\circ}$ (south), respectively $18^{\circ}$ (north) and $22^{\circ}$ (south) for cycle 23 , Makarov et al. 2002. They kept a spacing between them of somewhat more than $22^{\circ}$ (averages: $24.4^{\circ}$ north, $21.6^{\circ}$ south and $22.7^{\circ}$ for the spacing between the equatorial one (say the equator), slightly decreasing with the advancement of the cycles, i.e. at the approach of the equator. It is clear that the lower ones are getting too close (i.e. passing the more or less standard limiting spacing of $22^{\circ}$ ) to the equator, where the vague equatorial one splits to create a pair of new ones. These rest latitudes of the lower filament bands being too close to the equator may cause trouble (in volume and in gradient) to the differential rotation, responsible for amplification and/or generation of the magnetic fields. This may cause a deep minimum. The higher rest latitude is more or less the border of the sunspot butterfly diagram, and after its 'rest' it marks roughly the lower border of the polar faculae. The lower rest latitude is maybe associated with the two barely separated branches in each wing of the butterfly diagram. However, a clearer separation is marked by the polarity of the surges near sunspots. These surges are about 10-20 arcsec large and have on the higher side of the lower rest latitude still the same polarity sequence as the sunspots. $\mathrm{n}$ the lower side they have the opposite polarity sequence. 


\section{Conical blades of constant angular frequency}

We associate the higher 'rest latitudes' with the locus here the (internal) conical blades with vanishing radial gradient of the differential rotation cut the surface, Makarov et al. 2002, Callebaut, Makarov, Tlatov 2002. In those hyperboloidal blades the angular frequency corresponds to the one of the core which has no differential rotation. Of course the blades have not to be taken as mathematical surfaces, but as a region with quite a thickness in which the transition from $\partial_{r} \omega>0$ (sunspot region) to $\partial_{r} \omega<0$ (polar region) takes place. They are not static during a cycle as the convective zone is permanently in motion and as the amount of energy in the differential rotation varies (e.g. because the amplification of magnetic fields consumes some energy). Moreover, a region of transition $\left(\partial_{r} \omega=0\right)$ is easily subject to oscillations or even instabilities. From one cycle to next one the conical blades may come closer to the equator as more energy from the differential rotation is consumed. The region with $\partial_{r} \omega>0$ thus has narrowed, however, the gradient of the differential rotation may have increased (thus compensating for the smaller region); hence next cycle has not to be weaker. However, when the region becomes too narrow the cycle has to be small and this may lead to a deep minimum.

\section{Deep minimum approaching}

Several hints point in the same direction. Ribes \& Nesme-Ribes 1993 mention that during the Maunder Minimum the sunspots occurred very close to the equator and that the velocity gradients near the equator were very strong. Makarov et al. 2002 obtained from other arguments that the highest latitude of the upper rest latitude must be around $60^{\circ}$. Applying this value brings us back to the Dalton Minimum. The present anomaly in the Gnevyshev-Ohl rule of cycles 22-23 (Wolf numbers: 150 and 120 respectively, where one might have expected over 180 for the latter) is another sign. Moreover, according to the observations of the polar faculae and the polar magnetic field next cycle will attain $\mathrm{W}=70$ only. The polar faculae cycle showed a shift of $5.7 \pm 0.3$ year with the sunspot cycle for the cycles 20 to 22 ; for cycle 23 this shift amounts to 7.6 year.

\section{Conclusions}

We suggest that there is a connection between the upper rest latitudes $f$ the boundaries of the large-scale unipolar magnetic field regions (filament bands) and the hyperboloidal sheets with $\partial_{r} \omega=0$. There is some connection with the separation between the sunspot region and the polar faculae region too. The fact that the rest latitudes decreased steadily during the last 12 cycles approach the equator may indicate that a deep minimum is approaching. Other signals point in the same direction.

\section{Acknowledgements}

It is a pleasure to thank Dr. A. G. Tlatov for discussions.

\section{References}

Callebaut, D. K., Makarov, V. I. and Tlatov, A. G. 2002, Proc. 10th European Solar Physics Meeting, "Solar Variability: From Core to Outer Frontiers" ESA SP-506, 113.

Makarov, V. I., Tlatov, A. G., Callebaut, D. K., Obridko, V. N. 2002, Solar Phys. 206, 383.

Ribes, J. C., Nesme-Ribes, E. 1993, Astron. Ap. 276, 549. 